\title{
Dephasing of conduction electrons due to zero-point fluctuation
}

\author{
X. R. Wang \\ Department of Physics, Hong Kong University of Science and Technology, Hong Kong SAR, China \\ Gang Xiong \\ Department of Physics, Hong Kong University of Science and Technology, Hong Kong SAR, China \\ and Department of Physics, Wuhan University, Wuhan 430072, China \\ Shi-Dong Wang \\ Department of Physics, Hong Kong University of Science and Technology, Hong Kong SAR, China
}

(Received 2 November 1999)

\begin{abstract}
We consider conduction-electron dephasing by the zero-point fluctuation of an electromagnetic field in a vacuum. We argue that there is an upper cutoff frequency of the zero-point fluctuation mode beyond which the zero-point fluctuation will not contribute to the dephasing of conduction electrons. The upper cutoff frequency is material-parameter dependent. We find that the dephasing time is proportional to the electron diffusion constant. This result agrees very well with existing experimental data.
\end{abstract}

Electron dephasing is a very important subject in quantum systems. ${ }^{1-7}$ Tremendous debates ${ }^{5-9}$ have been concentrating on the possible zero-temperature dephasing of conduction electrons since Webb and his co-workers ${ }^{5,6}$ used zero-point fluctuation to explain their experiments. ${ }^{6}$ The standard weak localization theory predicts that the dephasing (decoherence or phase-relaxation) time (or length) of a conduction electron is infinite at zero temperature. However, Mohanty, Jariwala, and Webb (MJW) found that the dephasing time saturated at a finite value below $1 \mathrm{~K}$ in recent experiments on pure gold. ${ }^{6}$ In fact, saturated dephasing time (length) of conduction electrons was universally found in many early experiments. ${ }^{10-13}$ Those early experiments were explained by the heating or the magnetic impurity effect. The recent extensive experiments by MJW ruled out the popular extrinsic effects, ${ }^{10-13}$ and pointed toward the intrinsic dephasing of conduction electrons at zero temperature. ${ }^{5,6}$ This work has been drawing great attention in the condensed-matter physics community because there will be profound consequences if the proposal of zero-temperature dephasing is correct. Many results related to the Anderson localization need to be revised. ${ }^{4}$ According to the conventional scaling theory of localization, ${ }^{14}$ all states in a one-dimensional (1D) or a 2D system are localized if only scalar random potential is present. However, this statement is true only when the dephasing length (time) is infinite. Our current picture about metallic phase in low dimensions must be modified if there is an intrinsic zerotemperature dephasing mechanism such that the dephase length is intrinsically finite.

The center of the current debate is whether the saturation of the dephasing length (or time) at low temperature is due to an intrinsic zero-temperature dephasing mechanism or not. Golubev and Zaikin ${ }^{7}$ developed a detailed theory of zeropoint fluctuation which was originally proposed by MJW to explain their experiments. ${ }^{5}$ However, this theory was questioned. Altshuler, Gershenson, and Aleiner ${ }^{8}$ suggested external microwave as the dephasing source for the experiments. ${ }^{5}$ In a recent publication, Zawadowski, von Delft, and Ralph ${ }^{9}$ proposed a dynamical two-level system as the dephasing mechanism. The issue of zero-temperature dephasing remains controversial and inconclusive.

In this paper we consider the zero-temperature dephasing of conduction electrons due to the zero-point fluctuation of electromagnetic field (EMF) in a vacuum. The idea is similar to that in MJW's paper. ${ }^{6}$ The difference is in the source of the zero-point fluctuation. MJW considered the zero-point fluctuation from multidegrees of freedom of an electronic system due to the coupling or interaction among electrons rather than the more fundamental source of zero-point fluctuation of the EMF in a vacuum. The vacuum fluctuation of EMF is very important to many fundamental physics phenomena such as spontaneous emission and the Casimir force. ${ }^{15}$ The reason that zero-point fluctuation of EMF in a vacuum can dephase a conduction electron is because the EMF and electron cannot be separated, and electron motion is not a true eigenmode of the electron-EMF system. This is very much like electron levels in an atomic system. For example, a $2 p$ orbital in a hydrogen is not a true eigenmode of a hydrogen system because the electron in the hydrogen atom interacts with the EMF. Thus, the zero-point fluctuation of the EMF in a vacuum can induce an electron transition from $2 p$ orbital to $1 s$ orbital (the spontaneous emission).

So far, all experiments show universally that the saturated dephasing time at low temperature is proportional to the diffusion constant of conduction electrons. ${ }^{10-13}$ We believe that this overlooked experimental fact can be used to identify the true source of conduction-electron dephasing observed in experiments. $^{10-13}$

In order to study electron dephasing due to the vacuum fluctuation of the EMF, we start from the Aharonov-Bohm (AB) phase of an electron in an EMF

$$
\Psi(\vec{x})=\varphi(\vec{x}) \exp \left(i \frac{2 \pi}{\phi_{0}} \int \overrightarrow{\mathbf{A}} \cdot d l\right)
$$


where $\Psi$ is the electron wave function in the presence of vector potential $\overrightarrow{\mathbf{A}}, \varphi$ is the electron wave function in the absence of $\overrightarrow{\mathbf{A}}$, and $\phi_{0}=h / e$ is the flux quantum. This is a semiclassical approach because $\overrightarrow{\mathbf{A}}$ in this formulation is treated as a classic field while the electron is treated quantum mechanically. At zero temperature, though the average of $\overrightarrow{\mathbf{A}}$ is zero, the amplitude of $\overrightarrow{\mathbf{A}}$ would not always stay at zero even in the absence of an external EMF because of its zeropoint motion. It shall fluctuate randomly and independently in time and space. Therefore, a conduction electron can obtain a random phase when it diffuses from one point to another in a system because of the interaction between the electron and EMF through Eq. (1). The phase of the electron becomes completely random after a certain diffusion length.

What we need to evaluate is the ensemble average of the phase factor $\left\langle e^{i \phi}\right\rangle$ over the EMF zero-point fluctuation in a vacuum with the $\mathrm{AB}$ phase $\phi=\left(2 \pi / \phi_{0}\right) \int \overrightarrow{\mathrm{A}} \cdot \mathrm{d} l$. The conduction electron is dephased when this average value tends to zero due to the increasing destructive interference with the increase of the deviation of the $\mathrm{AB}$ phase. As the vacuum fluctuation of $\overrightarrow{\mathbf{A}}(\vec{r}, t)$ is random and independent both in time and space, phase $\phi$ is a random variable. For the Gaussian distribution of $\phi$, one has

$$
\left\langle e^{i \phi}\right\rangle=\exp \left(-\frac{1}{2}\left\langle\phi^{2}\right\rangle\right)
$$

where we have used the property that the average of $\phi$ is zero because the average of $\overrightarrow{\mathbf{A}}(\vec{r}, t)$ is zero for the vacuum fluctuation. $\left\langle\phi^{2}\right\rangle$ increases with the electron diffusion length. After some time it becomes much larger than 1, leading to $\left\langle e^{i \phi}\right\rangle \rightarrow 0$, i.e., the dephasing of the electron. Thus the dephasing time $\tau_{\phi}$ can be defined as the time when $\frac{1}{2}\left\langle\phi^{2}\left(\tau_{\phi}\right)\right\rangle \sim 1$.

Now we need to evaluate the mean-square deviation of the phase in the vacuum state

$$
\left\langle\phi^{2}\right\rangle=\frac{e^{2}}{\hbar^{2}}\left\langle\int d \vec{r}_{1} \cdot \overrightarrow{\mathbf{A}}\left[\vec{r}_{1}(t)\right] \int d \vec{r}_{2} \cdot \overrightarrow{\mathbf{A}}\left[\vec{r}_{2}\left(t^{\prime}\right)\right]\right\rangle,
$$

in which $\vec{r}(t)$ is the position vector of the conduction electron at time $t$. As a simple estimate, we replace the cosine factor of the inner product of $\overrightarrow{\mathbf{A}}$ and $d \vec{r}$ by its mean-square root value $\sqrt{\frac{1}{2} \int_{0}^{\pi} \sin \theta d \theta \cos ^{2} \theta}=\sqrt{1 / 3}$. Remember that vector potentials due to vacuum fluctuations at different space-time points do not correlate with each other, we obtain

$$
\left\langle\phi^{2}(\tau)\right\rangle \sim \frac{e^{2}}{3 \hbar^{2}} L(\tau)\left\langle\int d \vec{r} \overrightarrow{\mathbf{A}}^{2}[\vec{r}(t)]\right\rangle,
$$

where $L(\tau)=\sqrt{D \tau}$ is the diffusion distance of the electron in time $\tau$, and $D$ is the electron diffusion constant.

To evaluate the average of $\overrightarrow{\mathbf{A}}^{2}(\vec{r}, t)$ in vacuum state $|0\rangle$, we use its second-quantization representation ${ }^{15}$

$$
\overrightarrow{\mathbf{A}}(\vec{r}, t)=\sum_{\vec{k}, \lambda} \vec{\epsilon}_{\vec{k}, \lambda} \sqrt{\frac{2 \pi \hbar \mu_{0} c^{2}}{\omega_{k}}}\left[a_{\vec{k}} A_{0}(\vec{k}, t)+a_{\vec{k}}^{+} A_{0}^{*}(\vec{k}, t)\right],
$$

where $c$ is the speed of light, $\mu_{0}$ is permeability in a vacuum, $a_{\vec{k}}$ and $a_{\vec{k}}^{+}$are the annihilation and creation operator of a photon with wave number $\vec{k}, \vec{\epsilon}_{\vec{k}, \lambda}$ is the unit polarization vector of a mode with wave number $k$ and frequency $\omega_{k}$ $=k c$, and $A_{0}(\vec{k}, t)=e^{\left(i \vec{k} \cdot \vec{r}-i \omega_{k} t\right)}$. Substituting Eq. (5) into Eq. (4) and using $\left\langle 0\left|a_{\vec{k}_{1}} a_{\vec{k}_{2}}^{+}\right| 0\right\rangle=\delta_{\vec{k}_{1}, \vec{k}_{2}}$, we obtain

$$
\left\langle\phi^{2}(\tau)\right\rangle \sim \frac{8 \pi^{2} e^{2} \mu_{0} L^{2}(\tau)}{3 \hbar c} \int \omega d \omega,
$$

where $k=\omega / c$ is used and the integral includes frequencies of all modes which contribute to the AB phase in the time $\tau$.

As usual in quantum electrodynamics, an ultraviolet divergence appears in the vacuum fluctuation [Eq. (6)]. We need to introduce an upper cutoff frequency $\omega_{c}$ such that modes above this frequency should not contribute to the electron phase. In order to see whether such a cutoff frequency indeed exists, we notice that the $\mathrm{AB}$ phase is proportional to the convolution of vector potential $\overrightarrow{\mathbf{A}}$ along the diffusion path of electrons according to Eq. (1). In an extreme case when an electron does not move, the zero-point fluctuation of all EMF modes should not contribute to the electron phase because $d \vec{l}=0$. Now, if a conduction electron diffuses a distance $l$ during time $\tau$, modes which oscillate many times in $\tau$ should not contribute to the electron phase because the intergration over a fast mode is negligible. Therefore, the upper cutoff frequency $\omega_{c}$ is determined by the condition that an electron diffuses a distance $2 \pi c / \omega_{c}$ in a time of $2 \pi / \omega_{c}$, that is,

$$
\left(\frac{2 \pi c}{\omega_{c}}\right)^{2}=D \frac{2 \pi}{\omega_{c}}
$$

where $c$ is the speed of light and $D$ is the diffusion constant of electrons. Thus, the upper cutoff frequency is related to $D$ through $\omega_{c}=2 \pi c^{2} / D$. For a typical metal, $D \sim 10^{4} \mathrm{~m}^{2} / \mathrm{s}$, the cutoff frequency is about $10^{12}--10^{13} \mathrm{~Hz}$.

Substituting the upper cutoff frequency into Eq. (6), we obtain

$$
\left\langle\frac{\phi^{2}\left(\tau_{\phi}\right)}{2}\right\rangle \sim \frac{2 \pi^{3} e^{2} \mu_{0} c^{3} L_{\phi}^{2}}{3 D^{2} \hbar}=1,
$$

in which $L_{\phi}=\sqrt{D \tau_{\phi}}$ is the dephasing length. The dephasing time is then obtained as

$$
\tau_{\phi} \sim \frac{3 \hbar}{2 \pi^{3} \mu_{0} e^{2} c^{3}} D
$$

which shows that the dephasing time is proportional to the diffusion constant $D$ with a coefficiency of the order of $10^{-16} \mathrm{~s}^{2} / \mathrm{m}^{2}$. Taking $D \sim 10^{4} \mathrm{~m}^{2} / \mathrm{s}$, a typical value in metals, ${ }^{6}$ we obtain the dephasing time $\tau_{\phi} \sim 10^{-12} \mathrm{~s}$ which is comparable with the saturated dephasing time in experiments. ${ }^{6,10-12}$ It is worth while to point out that this dependence of the dephasing time on the electron diffusion constant, a material parameter, is from the upper cutoff frequency. Namely, the faster an electron diffuses, the less EMF modes can contribute to the electron $\mathrm{AB}$ phase. 
Before ending this paper, we would like to make two remarks. (1) EMF can influence electron motion not only through the $\mathrm{AB}$ phase but also through heating, magnetic confinement, etc. In the weak field such as that of a vacuum fluctuation, other effects can be neglected and the AB phase effect should dominate electron dephasing. For example, the strength of EMF in vacuum is about $\sqrt{2 \pi \hbar \mu_{0} \omega_{k}}$. The electron energy will change by $\Delta \epsilon=e \sqrt{2 \pi \hbar \mu_{0} \omega_{k}} l$ when an electron travels a distance of $l$ under the field. The electron will lose its phase after time $\tau$ when $\Delta \epsilon \tau=h$. Let $l^{2}=D \tau$, the dephasing time due to this mechanism is about $\left[2 \pi \hbar /\left(e^{2} \mu_{0} \omega_{k} D\right)\right]^{1 / 3}$. For $\omega_{k} \sim 10^{12} \mathrm{~Hz}, D \sim 10^{4} \mathrm{~m}^{2} / \mathrm{s}$, the typical time would be about $10^{-1} \mathrm{~s}$ which is much longer than that $\left({ }^{1} 0^{-12}\right.$ estimated in the previous section) from the $A B$ phase effect. (2) We assume that the vector potential $\overrightarrow{\mathbf{A}}$ is spatially uncorrelated for mathematical convenience. The real vacuum may not satisfy this assumption. The proportional constant in front of the diffusion constant $D$ in Eq. (9) should be modified for a real vacuum.

In summary, we propose a zero-temperature dephasing mechanism for conduction electrons based on zero-point fluctuation of EMF in a vacuum and the electron-EMF interaction through $\mathrm{AB}$ phase. We show that the dephasing time from this mechanism is proportional to the electron diffusion constant which coincides with previous experiments. ${ }^{6,10-12}$ This coincidence supports the idea that the saturation of dephasing time at the low temperature might be due to zeropoint fluctuation of EMF in a vacuum. There are a number of approximations in our calculation, such as the Gaussian distribution of the $\mathrm{AB}$ phase in vacuum. However, the physics that the zero-point fluctuation of EMF in a vacuum can dephase a conduction electron through $\mathrm{AB}$ phase should not depend on those approximations. A detailed theory beyond the semiclassical approach and approximations about the properties of EMF in a vacuum presented here would be interesting. Further experiments are needed to verify the importance of this mechanism to conduction electrons at low temperature.

One of us (X.R.W.) would like to thank Dr. Y. Q. Wang for stimulating discussions. This work was supported by UGC, Hong Kong, through RGC/DAG and RGC/CERG grants.
${ }^{1}$ B.L. Hu, J.P. Paz, and Y. Zhang, Phys. Rev. D 45, 2843 (1992).

${ }^{2}$ B.L. Hu et al., Int. J. Mod. Phys. A 10, 4537 (1995).

${ }^{3}$ W.H. Zurek, Phys. Today 44(10), 36 (1991).

${ }^{4}$ J. Shi, S. He, and X.C. Xie, cond-mat/9904393 (unpublished).

${ }^{5}$ P. Mohanty, E.M.Q. Jariwala, and R.A. Webb, Phys. Rev. Lett. 78, 3366 (1997).

${ }^{6}$ P. Mohanty and R.A. Webb, Phys. Rev. B 55, R13 452 (1997).

${ }^{7}$ D.S. Golubev and A.D. Zaikin, Phys. Rev. Lett. 81, 1074 (1998); cond-mat/9811185 (unpublished).

${ }^{8}$ B.L. Altshuler, M.E. Gershenson, and I.L. Aleiner, Physica E 3, 58 (1998).

${ }^{9}$ A. Zawadowski, Jan von Delft, and D.C. Ralph, Phys. Rev. Lett.
83, 2632 (1999)

${ }^{10}$ J.J. Lin and N. Giordano, Phys. Rev. B 35, 1071 (1987).

${ }^{11}$ D.M. Pooke, N. Paquin, M. Pepper, and A. Gundlach, J. Phys.: Condens. Matter 1, 3289 (1989).

${ }^{12}$ T. Hiramoto et al., Appl. Phys. Lett. 54, 2103 (1989).

${ }^{13}$ R.M. Mueller, R. Stasch, and G. Bergmann, Solid State Commun. 91, 255 (1994).

${ }^{14}$ E. Abahams, P.W. Anderson, D.C. Licciardello, and T.V. Ramakrishnan, Phys. Rev. Lett. 42, 673 (1979).

${ }^{15}$ Quantum Mechanics: A Modern Development, edited by Leslie E. Ballentina (World Scientific, Singapore, 1998). 